\title{
ATTENTIONAL TRADEOFFS IN THE PIGEON
}

\author{
O. V. Vyazovska, ${ }^{1}$ Y. Teng, ${ }^{2}$ ANd E. A. Wasserman ${ }^{2}$ \\ ${ }^{1}$ INSTITUTE FOR PROBLEMS OF CRYOBIOLOGY AND CRYOMEDICINE, NATIONAL ACADEMY OF SCIENCES OF UKRAINE \\ ${ }^{2}$ UNIVERSITY OF IOWA
}

\begin{abstract}
We deployed the Multiple Necessary Cues (MNC) discrimination task to see if pigeons can simultaneously attend to four different dimensions of complex visual stimuli. Specifically, we trained nine pigeons (Columba livia) on a go/no go discrimination to peck only 1 of 16 compound stimuli created from all possible combinations of two stimulus values from four separable visual dimensions: shape (circle/square), size (large/small), line orientation (horizontal/vertical), and brightness (dark/light). Some of the pigeons had CLHD (circle, large, horizontal, dark) as the positive stimulus $(\mathrm{S}+$ ), whereas others had SSVL (square, small, vertical, light) as the $\mathrm{S}+$. We recorded touchscreen pecking during the first $15 \mathrm{~s}$ that each stimulus was presented on each training trial. Discrimination training continued until pigeons' rates of responding to all 15 negative stimuli (S-s) fell to less than $15 \%$ of their response rates to the $\mathrm{S}+$. All pigeons acquired the MNC discrimination, suggesting that they attended to all four dimensions of the multidimensional stimuli. Learning rate was similar for all four dimensions, indicating equivalent salience of the discriminative stimuli. The more dimensions along which the $\mathrm{S}$-s differed from the $\mathrm{S}+$, the faster was discrimination learning, suggesting an added benefit from increasing perceptual disparities of the S-s from the $\mathrm{S}+$. Finally, evidence of attentional tradeoffs among the four dimensions was seen during discrimination learning, raising interesting questions concerning the possible control of behavior by elemental and configural stimuli.

Key words: discrimination learning, attentional tradeoff, dimensional stimulus control, configural stimulus control, vision, touchscreen pecking, pigeon
\end{abstract}

Over 50 years ago, Reynolds (1961) provided perhaps the clearest behavioral definition of attention ever offered. He did so in connection with a pioneering investigation into pigeons' learning visual discriminations, in which redundant relevant cues were associated with reinforced or nonreinforced key pecking. Reynolds proposed that, "an organism attends to an aspect of the environment if independent variation or independent elimination of that

We would like to thank Esther Mondragón (and her colleagues) in the Centre for Computational and Animal Learning Research for prompt and helpful consultations and for making the R\&W Simulator so readily available. We also thank D. V. Pavlenko in the Department of Physical and Biomedical Electronics and Complex Information Technologies, Radiophysics faculty, V. N. Karazin Kharkiv National University, O. P. Pryhod'ko, Associate Professor in the Department of Mathematical Modeling, Mechanics and Mathematics faculty, V. N. Karazin Kharkiv National University, and D. A. Shelkovenkov, Ph.D., Radiolocation and Radionavigation engineer at E.P.S. Ltd., for their technical support. Additional thanks go to Leyre Castro in the Department of Psychology, University of Iowa for her help in the conduct and reporting of this research.

Correspondence concerning this article should be addressed to Edward A. Wasserman, Department of Psychology, The University of Iowa, Iowa City, IA 52242. Email: ed-wasserman@uiowa.edu

doi: $10.1002 /$ jeab.82 aspect brings about variation in the organism's behavior" (p. 203).

As to the specific results of Reynolds' (1961) experiments, they showed "that a pigeon may attend to only one of several aspects of a discriminative stimulus. Every part of the environment that is present when a reinforced response occurs may not subsequently be an occasion for the emission of that response" (p. 208). Therefore, according to Reynolds, "attention refers to the controlling relation between a stimulus and responding. An organism attends to a stimulus when its responding is under the control of that stimulus" (p. 208).

Subsequent to Reynolds' innovative research and incisive behavioral analysis (unfortunately ignored in Lovie's 1983 historical consideration of attention and behaviorism), it has become standard practice to use the Redundant Relevant Cues (RRC) task to study attention in nonhuman animals, as in the later work of Johnson and Cumming (1968), Farthing and Hearst (1970), and Wilkie and Masson (1976). It has also been standard practice to study just two dimensions of compound discriminative stimuli. Finally, because of the nature of RRC tasks, tests for stimulus control have followed successful discrimination mastery. 
Nevertheless, the RRC task is not the only discrimination learning method that can be effectively deployed in the behavioral study of attention in animals. Our laboratory developed the Multiple Necessary Cues (MNC) task over 20 years ago in the hope that it might provide fresh insight into how animals attend to even more complex discriminative stimuli, and that it might be able to do so while learning is transpiring rather than after the task has already been acquired (Chatlosh \& Wasserman, 1993; Gottselig, Wasserman, \& Young, 2001; Soto \& Wasserman, 2010, 2011; also see Blough \& Blough, 1997 for other highly effective behavioral methods for studying attention in animals). In the present study, we report the results of our most comprehensive effort yet to explore the unfolding of attention in pigeons.

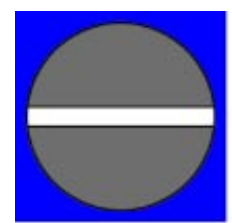

CLHD

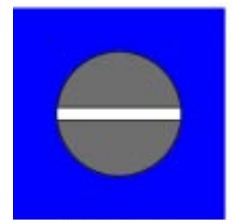

CSHD

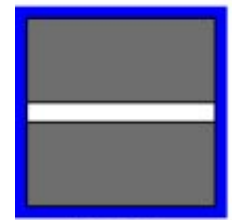

SLHD

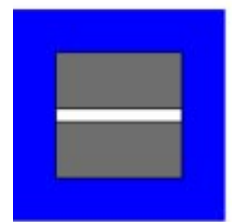

SSHD

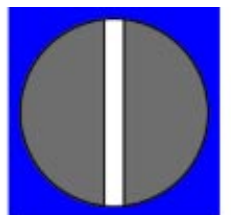

CLVD

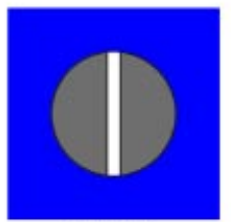

CSVD

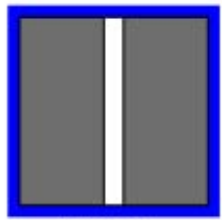

SLVD

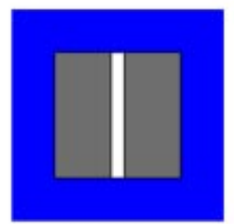

SSVD

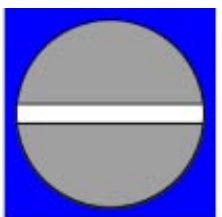

CLHL

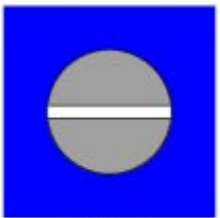

CSHL

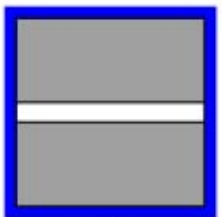

SLHL

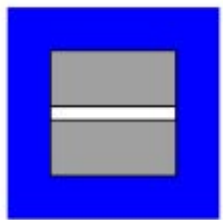

SSHL

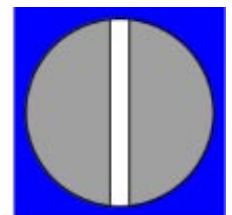

CLVL

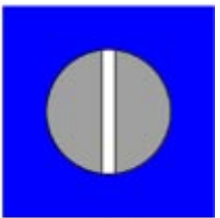

CSVL

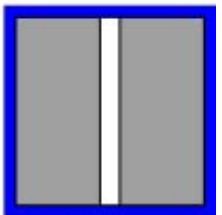

SLVL

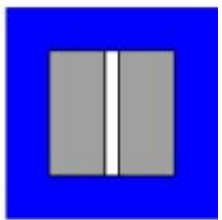

SSVL

All 16 discriminative stimuli created from two possible values along four dimensions: Circle/Square, Large/Small, Horizontal Line/Vertical Line, and Dark/Light. Surrounding the discriminative stimuli and occupying the full LCD display was a Blue field. Shown is only that portion of the Blue field (the darker bordering region) that fell within the effective pecking area containing each discriminative stimulus. That area was slightly larger $(6.4 \mathrm{~cm})$ than the large circle and square stimuli themselves $(5.6 \mathrm{~cm})$, but was the same for both the large $(5.6 \mathrm{~cm})$ and the small $(3.8 \mathrm{~cm})$ stimuli. 
Successful discrimination performance would be consistent with the pigeons' having attended to all four dimensions of the multidimensional discriminative stimuli. More importantly, monitoring discriminative responding to each separate dimension of the stimuli on each day during discrimination training might provide particularly important evidence as to: (1) the differential salience of the four visual dimensions, (2) the possibly increasing discriminability of the 15 $\mathrm{S}$-s the more dimensional disparities that distinguished each $\mathrm{S}_{-}$- from the $\mathrm{S}+$, and (3) the possibility that the four-dimensional discrimination might prove to be sufficiently demanding for pigeons that tradeoffs in attention might be seen throughout the course of discrimination learning, with enhanced discriminative performance to one dimension coming at the expense of discriminative performance to one or more of the other dimensions.

Such "attentional tradeoffs" are believed to be the consequence of two fundamental and logically related aspects of attention (Pashler, 1998): limited capacity and selectivity. If an animal's attentional capacities are overloaded, then selectivity is a necessary consequence of limited capacity. The notion that paying more attention to some discriminative stimuli causes the loss of attention to others is called the "inverse hypothesis" (Thomas, 1970).

Most theories of selective attention in discrimination learning have assumed the inverse hypothesis to be true (Kruschke \& Johansen, 1999; Riley \& Roitblat, 1978; Sutherland \& Mackintosh, 1971; Trabasso \& Bower, 1968). For example, Sutherland and Mackintosh's highly influential theory proposed that animals learn to attend to the relevant dimension(s) of a stimulus using dimensional analyzers whose strengths sum to a constant amount; when one analyzer is strengthened, the total strengths of the other analyzers must be weakened by the same amount (Sutherland \& Mackintosh, 1971). Selective attention has further been hypothesized to be a "central adaptation to information overload” (Riley \& Roitblat, p. 249).

Of course, such a dimensional attentional analysis might not provide a full or even accurate account of the pigeons' discrimination behavior (for more on the empirical status of the inverse hypothesis, see Gottselig et al., 2001)configural stimuli may also play a critical part (e.g., Pearce, 2002). Detailed scrutiny and mathematical modeling of both overall and daily discrimi- nation behavior in our study provided clues as to the possible participation of dimensional and configural stimuli in MNC discrimination learning.

\section{Method}

\section{Subjects}

A total of nine experimentally naive adult pigeons (Columba livia) of mixed sex were studied; they were kept in individual $50 \mathrm{~cm} \times 50 \mathrm{~cm}$ cages under a 12:12 hour light/dark cycle (lights on at 8:00 AM). Throughout the experiment, the pigeons were maintained at $85 \%$ of their freefeeding weights, with water freely available in the home cages.

\section{Apparatus}

The pigeons were trained in a standard operant conditioning chamber $(35 \times 35 \times 35 \mathrm{~cm})$. The front wall of the chamber contained a transparent rectangular frame $(9.50 \mathrm{~cm}$ high $\times 17.50 \mathrm{~cm}$ wide) made of Plexiglas. The bottom edge of the frame was $15.50 \mathrm{~cm}$ above the floor; the left and right edges were equidistant from the side walls. Immediately behind the frame was a resistive touchscreen; pecks to the touchscreen were limited to the opening in the rectangular frame and were processed by a controller board outside the chamber. A TFT LCD display was located $2 \mathrm{~mm}$ behind the touchscreen. A food delivery cup was placed in the floor directly beneath the touchscreen opening; mixed grain reinforcers could be dispensed into this cup via a rotary delivery mechanism.

During experimental sessions, the chamber was constantly illuminated by a $3.5-\mathrm{V}$ incandescent lamp located near the top of the rear wall. A PC running Matlab $10 \mathrm{~b}^{\circledR}$ software controlled discriminative stimulus presentation, the delivery of food reinforcement, and the recording of touchscreen responses.

\section{Discriminative Stimuli}

We prepared a total of 16 different Shape/ Size/Orientation/Brightness compound visual stimuli created from two possible values along four dimensions: Circle/Square, Large/Small, Horizontal Line/Vertical Line, and Dark/Light (Fig. 1). These dimensions were chosen because of their proven effectiveness (Castro \& Wasserman, 2013; Gottselig et al., 2001) and their separability (for more on the role of 
separability and integrality in pigeons' visual discrimination behavior, see Soto \& Wasserman, 2010).

The diameter of the Large Circle and the width of the Large Square was $5.60 \mathrm{~cm}$; the diameter of the Small Circle and the width of the Small Square was $3.80 \mathrm{~cm}$. The width of the line with the Large Circle and the Large Square was $0.65 \mathrm{~cm}$; the width of the line with the Small Circle and the Small Square was $0.45 \mathrm{~cm}$. The RGB values of the Dark stimuli were 110, 110, 110; the RGB valued of the Light stimuli were $160,160,160$. The discriminative stimuli were presented in the center of the touchscreen frame on a blue field (RGB values were $0,0,255$ ) which filled the entire LCD display. The effective pecking area containing each discriminative stimulus (Large or Small) was $6.40 \times 6.40 \mathrm{~cm}$ in order to equate the opportunity to record pecks from stimuli of both Large and Small sizes.

\section{Procedure}

Shaping. Because the pigeons were experimentally naïve, they first had to be trained to eat from the food cup and to peck the touchscreen. We thus conducted manual shaping with the same discriminative stimuli as would later be used in Baseline and Discrimination Training.

Baseline Training. Next, the pigeons began Baseline Training, during which pecking all 16 discriminative stimuli was reinforced on a Fixed Interval 15-s schedule. Daily sessions contained 10 blocks of trials involving all 16 of the discriminative stimuli for a total of 160 trials; stimulus presentation was randomized within blocks (this method of trial presentation was the same as was used during later Discrimination Training). On each trial, the discriminative stimulus remained on the screen for at least $15 \mathrm{~s}$; the first peck after $15 \mathrm{~s}$ turned off the stimulus and delivered 1 to $2 \mathrm{~s}$ of food. Intertrial intervals (ITIs) were randomized between 6 and $10 \mathrm{~s}$; during ITIs, the houselight remained illuminated. Each pigeon received Baseline Training until its daily response rate to each discriminative stimulus was within $20 \%$ of its mean rate to all 16 discriminative stimuli.

Discrimination Training. Finally, the pigeons were moved to Discrimination Training. Four pigeons were given SSVL as the $\mathrm{S}+$; the other five pigeons were given CLHD as the $\mathrm{S}+$. $\mathrm{On} \mathrm{S}+$ trials, the discriminative stimulus remained on the screen for at least $15 \mathrm{~s}$; the first peck after $15 \mathrm{~s}$ turned off the stimulus and delivered 5 to $6 \mathrm{~s}$ of food. (This longer duration of food delivery allowed us to maintain the birds' weights at the $85 \%$ level, given that only 1 out of 16 trials was now scheduled to involve food reinforcement.) On S- trials, the discriminative stimulus remained on the screen for $15 \mathrm{~s}$, immediately followed by the ITI. In order to equate the opportunity for responding to count on $\mathrm{S}+$ and S- trials, only those pecks that occurred during the first $15 \mathrm{~s}$ of stimulus presentation were recorded and analyzed. The criterion for completing Discrimination Training was that, in a single daily session, the bird's rate of response (pecks per s) to each of the $15 \mathrm{~S}$-s was less than $15 \%$ of its rate to $\mathrm{S}+$. (That criterion was slightly relaxed for P8, which met an $18 \%$ criterion in 34 days, 1 day longer than any other bird in the experiment).

\section{Results}

The MNC task affords the experimental subject a variety of different routes to ultimate discrimination mastery. In the current experiment-involving four dimensional elements and their possible configurations-the analysis of our pigeons' behavior over time presented many analytical and interpretive challenges. We correspondingly deployed several different methods to capture and comprehend behavioral trends of considerable complexity.

\section{Discrimination Learning}

All nine pigeons eventually learned the fourdimensional discrimination task, although there were considerable individual differences in the speed of learning among the birds. Response rates to all $15 \mathrm{~S}$-s did succeed in falling below $15 \%$ of the response rate to the $\mathrm{S}+$ in a single session within 5 to 34 days of Discrimination Training (18\% in 34 days in the case of P8).

Table 1 shows the mean rate of response for each pigeon to all 16 discriminative stimuli up to and including the criterion day of Discrimination Training. Table 2 shows the mean rate of response for each pigeon to all 16 discriminative stimuli on the criterion day of Discrimination Training. Comparing the scores in Tables 1 and 2 indicates that changes in response rates to the S-s were primarily responsible for discrimination 
Table 1

Mean Response Rate Across All Days of Discrimination Training

\begin{tabular}{|c|c|c|c|c|c|c|c|c|c|}
\hline \multirow[b]{4}{*}{ Stimulus } & \multicolumn{9}{|c|}{ Response Rate (Pecks/s) } \\
\hline & \multicolumn{9}{|c|}{ Bird (Days of Training) } \\
\hline & \multicolumn{5}{|c|}{ CLHD+ Condition } & \multicolumn{4}{|c|}{ SSVL+ Condition } \\
\hline & P20 (7) & P21 (15) & P10 (20) & P17 (23) & P19 (33) & P9 (5) & P7 (9) & P11 (9) & P8 (34) \\
\hline CLHD & 1.45 & 2.59 & 1.32 & 2.69 & 3.08 & 0.13 & 0.14 & 0.19 & 0.02 \\
\hline CLHL & 0.71 & 0.80 & 0.08 & 0.29 & 0.53 & 0.31 & 0.20 & 0.23 & 0.03 \\
\hline CLVD & 0.14 & 0.45 & 0.07 & 0.25 & 0.34 & 0.24 & 0.23 & 0.25 & 0.07 \\
\hline CLVL & 0.11 & 0.26 & 0.01 & 0.03 & 0.07 & 0.43 & 0.23 & 0.38 & 0.20 \\
\hline CSHD & 0.60 & 1.46 & 0.46 & 1.48 & 1.19 & 0.19 & 0.16 & 0.27 & 0.03 \\
\hline CSHL & 0.17 & 0.37 & 0.02 & 0.11 & 0.13 & 0.29 & 0.23 & 0.35 & 0.07 \\
\hline CSVD & 0.12 & 0.31 & 0.02 & 0.12 & 0.07 & 0.38 & 0.23 & 0.42 & 0.21 \\
\hline CSVL & 0.06 & 0.15 & 0.01 & 0.03 & 0.05 & 0.61 & 0.41 & 0.90 & 0.60 \\
\hline SLHD & 0.38 & 1.64 & 0.45 & 1.12 & 0.70 & 0.25 & 0.24 & 0.27 & 0.03 \\
\hline SLHL & 0.20 & 0.39 & 0.03 & 0.14 & 0.08 & 0.39 & 0.45 & 0.65 & 0.20 \\
\hline SLVD & 0.07 & 0.36 & 0.02 & 0.09 & 0.05 & 0.39 & 0.44 & 0.52 & 0.09 \\
\hline SLVL & 0.12 & 0.19 & 0.01 & 0.06 & 0.03 & 0.65 & 0.76 & 1.23 & 0.54 \\
\hline SSHD & 0.24 & 0.78 & 0.08 & 0.95 & 0.21 & 0.26 & 0.34 & 0.52 & 0.18 \\
\hline SSHL & 0.13 & 0.22 & 0.01 & 0.13 & 0.05 & 0.58 & 0.90 & 1.38 & 1.34 \\
\hline SSVD & 0.13 & 0.14 & 0.01 & 0.09 & 0.06 & 0.65 & 0.63 & 1.60 & 0.96 \\
\hline SSVL & 0.12 & 0.11 & 0.01 & 0.04 & 0.01 & 1.73 & 1.46 & 2.56 & 2.25 \\
\hline
\end{tabular}

Note. Response rates tso the $\mathrm{S}+$ compound are in bold.

learning; changes in response rates to the $\mathrm{S}+\quad$ behavior despite the requirement that attention were not consistently associated with the unfold- be paid to all four separate aspects of the ing of discrimination learning.

Clearly, MNC training proved to be extremely effective in supporting highly discriminative attentional limit of pigeons.

Table 2

Mean Response Rate on Final Day of Discrimination Training

\begin{tabular}{|c|c|c|c|c|c|c|c|c|c|}
\hline \multirow[b]{4}{*}{ Stimulus } & \multicolumn{9}{|c|}{ Response Rate (Pecks per s) } \\
\hline & \multicolumn{9}{|c|}{ Bird (Days of Training) } \\
\hline & \multicolumn{5}{|c|}{ CLHD+ Condition } & \multicolumn{4}{|c|}{ SSVL+ Condition } \\
\hline & P20 (7) & P21 (15) & P10 (20) & P17 (23) & P19 (33) & P9 (5) & P7 (9) & P11 (9) & P8 (34) \\
\hline CLHD & 1.45 & 2.68 & 1.94 & 2.75 & 2.67 & 0.00 & 0.00 & 0.01 & 0.01 \\
\hline CLHL & 0.09 & 0.14 & 0.00 & 0.01 & 0.05 & 0.00 & 0.02 & 0.02 & 0.02 \\
\hline CLVD & 0.05 & 0.02 & 0.00 & 0.22 & 0.02 & 0.00 & 0.00 & 0.00 & 0.11 \\
\hline CLVL & 0.00 & 0.01 & 0.00 & 0.00 & 0.00 & 0.02 & 0.04 & 0.04 & 0.09 \\
\hline CSHD & 0.12 & 0.24 & 0.25 & 0.15 & 0.09 & 0.00 & 0.03 & 0.04 & 0.03 \\
\hline CSHL & 0.01 & 0.01 & 0.00 & 0.00 & 0.00 & 0.00 & 0.01 & 0.03 & 0.04 \\
\hline CSVD & 0.00 & 0.00 & 0.00 & 0.02 & 0.00 & 0.01 & 0.03 & 0.06 & 0.13 \\
\hline CSVL & 0.00 & 0.03 & 0.00 & 0.00 & 0.00 & 0.09 & 0.08 & 0.09 & 0.37 \\
\hline SLHD & 0.04 & 0.31 & 0.24 & 0.09 & 0.14 & 0.01 & 0.03 & 0.01 & 0.01 \\
\hline SLHL & 0.03 & 0.07 & 0.00 & 0.00 & 0.00 & 0.05 & 0.04 & 0.13 & 0.07 \\
\hline SLVD & 0.06 & 0.15 & 0.00 & 0.01 & 0.00 & 0.03 & 0.02 & 0.01 & 0.04 \\
\hline SLVL & 0.00 & 0.00 & 0.00 & 0.00 & 0.00 & 0.09 & 0.20 & 0.29 & 0.25 \\
\hline SSHD & 0.03 & 0.11 & 0.03 & 0.03 & 0.03 & 0.02 & 0.03 & 0.03 & 0.03 \\
\hline SSHL & 0.00 & 0.00 & 0.00 & 0.00 & 0.00 & 0.11 & 0.23 & 0.28 & 0.09 \\
\hline SSVD & 0.00 & 0.07 & 0.00 & 0.00 & 0.00 & 0.13 & 0.03 & 0.09 & 0.05 \\
\hline SSVL & 0.00 & 0.01 & 0.00 & 0.00 & 0.00 & 1.64 & 1.67 & 2.17 & 2.06 \\
\hline
\end{tabular}

Note. Response rates to the $\mathrm{S}+$ compound are in bold. 


\section{Dimensional Stimulus Control}

We next explored whether the four visual dimensions differentially controlled the pigeons' responding. To find out, we examined the mean rates of response to each of the four dimensions for each pigeon across all of its Discrimination Training sessions. Specifically, two response rate scores were computed for each of the four dimensions: one for the $\mathrm{S}+$ value (for example, the Circle) and one for the $\mathrm{S}-$ value (for example, the Square). From those two response rates (for example, one calculated from the eight compound stimuli containing the Circle, the S+ value, and the second calculated from the eight compound stimuli containing the Square, the Svalue), we calculated a Discrimination Ratio (DR) score for each of the four dimensions: $8 \mathrm{~S}+$ compounds $\div[(8 \mathrm{~S}+$ compounds $)+(8 \mathrm{~S}-$ compounds)]. We then determined whether there were decidedly different DRs for each dimension for each pigeon and for the entire contingent of birds. These scores are shown in Table 3.

In general, there were small disparities in discriminative responding to the four visual dimensions. Individual pigeons' DRs across all days of Discrimination Training ranged from .65 to .93 , with individual birds' lowest to highest DR disparities among the four dimensions ranging from .05 to .26 . Overall DRs among the four dimensions varied from .73 to .81 - a range of only .08. We statistically assessed possible mean differences in salience among the four dimensions with a one-way Analysis of Variance (ANOVA). The main effect of Dimension was not statistically significant, $F(3,24)=1.85, p>.05$. Thus, the four visual dimensions did not differentially control the pigeons' responding.

\section{Control by Number of Dimensional Disparities}

The 15 S-s might have differentially controlled the pigeons' responding depending on how discrepant each $\mathrm{S}$ - was from the $\mathrm{S}+$ (Pearce, 2002). To explore this matter, we grouped the S-s in accord with the number of ways in which each differed from the $\mathrm{S}+$ : by four dimensional disparities (DD4), by three dimensional disparities (DD3), by two dimensional disparities (DD2), and by only one dimensional disparity (DD1). For example, with the CLHD $\mathrm{S}+$, the four DD1 S-s were CLHL, CLVD, CSHD, and SLHD; the six DD2 S-s were CLVL, CSHL, CSVD, SLHL, SLVD, and SSHD; the four DD3 Ss were CSVL, SLVL, SSHL, and SSVD; and, the one DD4 S- was SSVL. Then, we calculated the mean response rate to these four different types of S- and the mean rates of response to the remaining complements of $\mathrm{S}+$ stimuli $(12 \mathrm{~S}+$ compounds in the case of DD1,10S+ compounds in the case of DD2, $12 \mathrm{~S}+$ compounds in the case of DD3, and $15 \mathrm{~S}+$ compound in the case of DD4) yielding four different DRs: $(\mathrm{S}+$ compounds) $\div[(\mathrm{S}+$ compounds $)+(\mathrm{S}-$ compounds $)]$. These dimensional discrepancy scores are shown in Table 4.

Overall DRs were ordered DD4 $>$ DD3 $>$ DD2 $>$ DD1. All nine pigeons exhibited the lowest DR on DD1 trials and the second lowest DR on DD2 trials. We statistically assessed possible differences in performance among the four dimensional disparities with a oneway ANOVA. The main effect of Dimensional Disparity was statistically significant, $F(3$, $24)=39.02, p<.001$. Tukey follow-up tests disclosed that discriminative performance on DD1 trials was significantly lower than on

Table 3

Mean Discrimination Ratios for Each Different Dimension

\begin{tabular}{|c|c|c|c|c|c|c|c|c|c|c|}
\hline \multirow[b]{4}{*}{ Dimension } & \multicolumn{10}{|c|}{ Discrimination Ratio: $(\mathrm{S}+$ compounds $) /(\mathrm{S}+$ compounds $+\mathrm{S}-$ compounds $)$} \\
\hline & \multicolumn{10}{|c|}{ Bird (Days of Training) } \\
\hline & \multicolumn{5}{|c|}{ CLHD+ Condition } & \multicolumn{4}{|c|}{ SSVL+ Condition } & \multirow[b]{2}{*}{ Mean } \\
\hline & P20 (7) & P21 (15) & P10 (20) & P17 (23) & P19 (33) & P9 (5) & P7 (9) & P11 (9) & P8 (34) & \\
\hline Orientation & 0.89 & 0.84 & 0.93 & 0.92 & 0.93 & 0.73 & 0.65 & 0.71 & 0.71 & 0.81 \\
\hline Brightness & 0.75 & 0.78 & 0.92 & 0.92 & 0.89 & 0.72 & 0.75 & 0.74 & 0.78 & 0.80 \\
\hline Shape & 0.79 & 0.66 & 0.75 & 0.72 & 0.85 & 0.73 & 0.81 & 0.82 & 0.84 & 0.77 \\
\hline Size & 0.75 & 0.70 & 0.77 & 0.66 & 0.76 & 0.68 & 0.69 & 0.75 & 0.83 & 0.73 \\
\hline
\end{tabular}


Table 4

Mean Discrimination Ratios Across All Days of Discrimination Training for Different Numbers of Dimensional Disparities (DDs)

\begin{tabular}{|c|c|c|c|c|c|c|c|c|c|c|}
\hline \multirow{4}{*}{$\begin{array}{l}\text { Number } \\
\text { of DDs }\end{array}$} & \multicolumn{10}{|c|}{ Discrimination Ratios: $(\mathrm{S}+$ compounds $) /(\mathrm{S}+$ compounds $+\mathrm{S}-$ compounds $)$} \\
\hline & \multicolumn{10}{|c|}{ Bird (Days of Training) } \\
\hline & \multicolumn{5}{|c|}{ CLHD+ Condition } & \multicolumn{4}{|c|}{ SSVL+ Condition } & \multirow[b]{2}{*}{ Mean } \\
\hline & P20 (7) & P21 (15) & $\mathrm{P} 10(20)$ & P17 (23) & P19 (33) & P9 (5) & P7 (9) & P11 (9) & P8 (34) & \\
\hline One & 0.78 & 0.71 & 0.80 & 0.77 & 0.82 & 0.73 & 0.70 & 0.70 & 0.71 & 0.75 \\
\hline Two & 0.91 & 0.86 & 0.96 & 0.91 & 0.96 & 0.81 & 0.84 & 0.86 & 0.92 & 0.89 \\
\hline Three & 0.94 & 0.92 & 0.98 & 0.96 & 0.98 & 0.85 & 0.88 & 0.92 & 0.97 & 0.93 \\
\hline Four & 0.93 & 0.95 & 0.98 & 0.97 & 0.99 & 0.90 & 0.92 & 0.94 & 0.97 & 0.95 \\
\hline
\end{tabular}

DD2, DD3, and DD4 trials (all $p \mathrm{~s}<.05$ ); in addition, discriminative performance on DD2 trials was significantly lower than on DD4 trials $(p<.05)$.

\section{Attentional Tradeoffs: Discrimination Ratios}

We next considered individual pigeons' dimensional DRs throughout the course of Discrimination Training. For illustrative purposes, we chose six focal birds which displayed what appeared by inspection to be few, moderate, or many coordinated reversals between the visual dimensions during discrimination learning (the behavior of the three remaining pigeons is depicted in the Supplementary Electronic Material); in each case, one bird was chosen from each S+ condition (SSVL and CLHD). These scores are illustrated in Figures 2-4. Upswings and downswings in dimensional discrimination performance were sometimes small (Fig. 2), sometimes large (Fig. 4), and often associated with one another (Figs. 3 and 4), consistent with attentional tradeoffs.

Beginning with the pair of birds shown in Figure 4, P19 appeared to be acquiring all four dimensional discriminations quite proficiently until Day 6, when further increases in the Orientation, Brightness, and Shape discriminations were associated with a 3-day decline in the Size discrimination. When the Size discrimination rebounded on Day 9, there was a dramatic drop in the Shape discrimination and a smaller drop in the Orientation discrimination. Subsequent Size-Shape tradeoffs can be seen on Days 17,21 , and 25. Several other interdimensional tradeoffs are more difficult to discern in Figure 4 - such as between Orientation and the other three dimensions on Days 12 and 13-because of the many peaks and valleys in this pigeon's DRs. P8, too, seemed to be proficiently acquiring all four dimensional discriminations until Day 5 , when the Orientation discrimination fell. The Brightness and Shape DRs subsequently fell as the Orientation discrimination rebounded on Days 6 and 7. Days 16, 19, and 21 mark other occasions on which the Brightness and Orientation DRs moved in opposite directions. Days 25 to 27 proved to be a particularly interesting period: here, an abrupt rise in the Orientation DR was accompanied by sharp falls in the Shape, Brightness, and Size DRs. Indeed, until Day 25, Shape had generally been the best discriminated dimension; however, thereafter, it was generally the poorest discriminated dimension.

Moving next to the pair of birds shown in Figure 3, for P21, from Day 4 to Day 5, the Brightness DR rose, but the Size DR fell. From Day 5 to Day 6, the Shape DR went up, but the Brightness DR went down. From Day 6 to Day 8, the Size DR increased, but the Orientation DR decreased. Finally, from Day 12 to Day 13, the Size DR rose, but the Brightness DR fell. For P7, from Day 3 to Day 4, the Brightness DR went up, but the Orientation DR went down. And, from Day 4 to Day 5 , the Size DR rose, but the Orientation DR fell.

And, considering the pair of birds shown in Figure 2, for P20, from Day 3 to Day 5, both Brightness and Shape DRs increased, but the Size DR decreased. For P9, from Day 3 to Day 5 , both Brightness and Size DRs went up, whereas the Orientation DR went down.

We tried many different ways to quantify what to the eye appeared to be attentional tradeoffs, with varying degrees of success. In the relatively simple method reported here, we calculated pairwise Pearson correlations among all four 


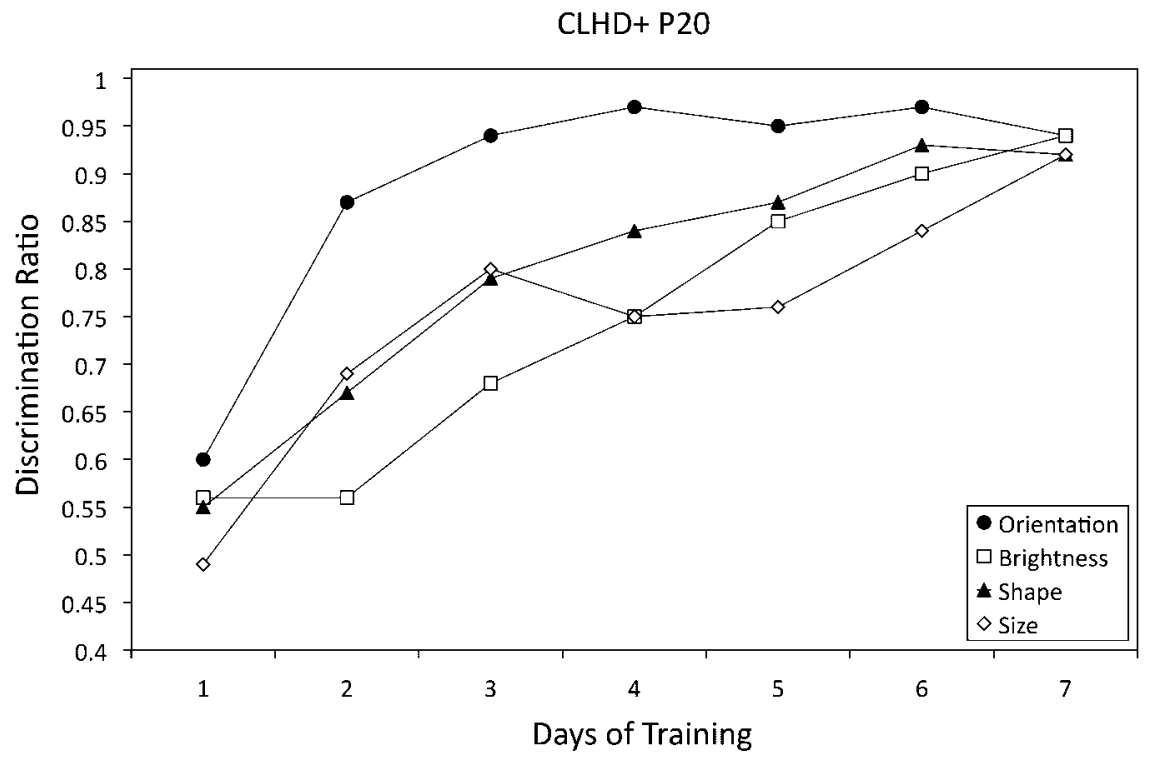

SSVL+ P9

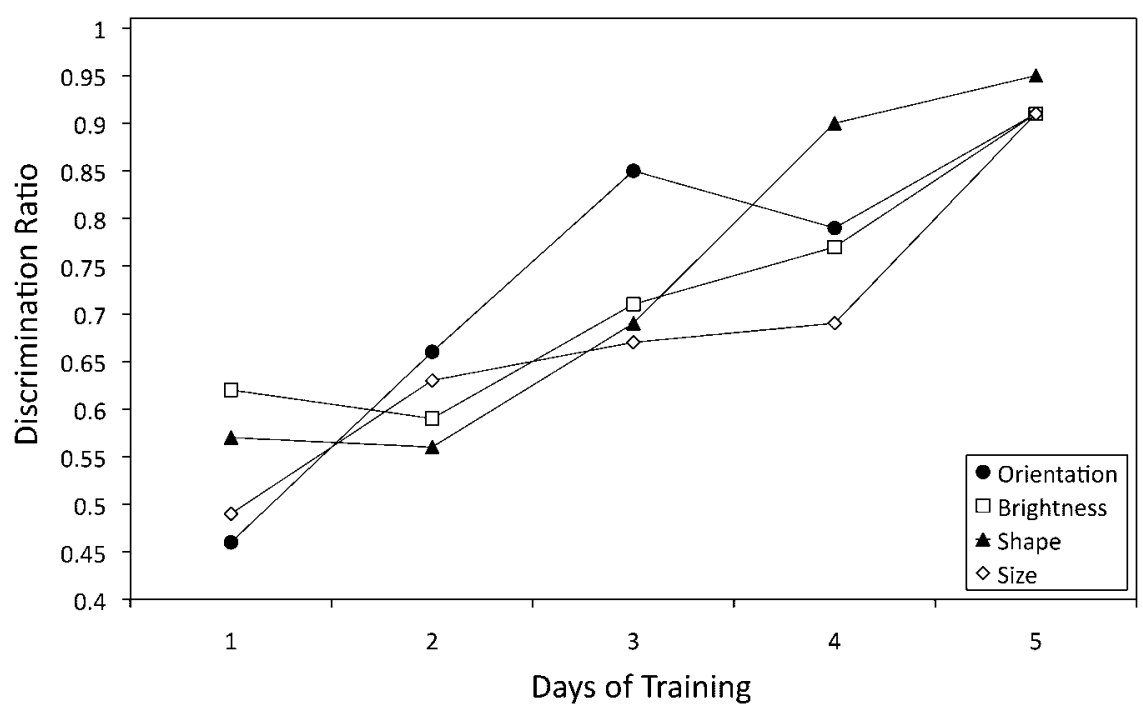

Fig. 2. Discrimination Ratios (DRs) for Shape, Size, Orientation, and Brightness for P20 (top) and P9 (bottom) throughout the entirety of Discrimination Training.

dimensional DRs derived from each of the nine birds' daily responding to all 16 discriminative stimuli. Because the pigeons were generally evidencing increasing DRs as they acquired the four-dimensional discrimination, such increases ought to have been very highly correlated with one another. On the other hand, relatively low correlations between paired dimensional DRs would be consistent with the presence of attentional tradeoffs. Table 5 shows that $61 \%$
$(33 / 54)$ of the correlations were at or below .75 and $22 \%(12 / 54)$ of the correlations were at or below .50.

Such correlational evidence may well suggest the occurrence of attentional tradeoffs, but that evidence may not be dispositive. One problem with this analytical approach is that it is difficult to say just how low or high these paired dimensional correlations ought to be before we can confidently conclude that attentional 
$\mathrm{CLHD}+\mathrm{P} 21$

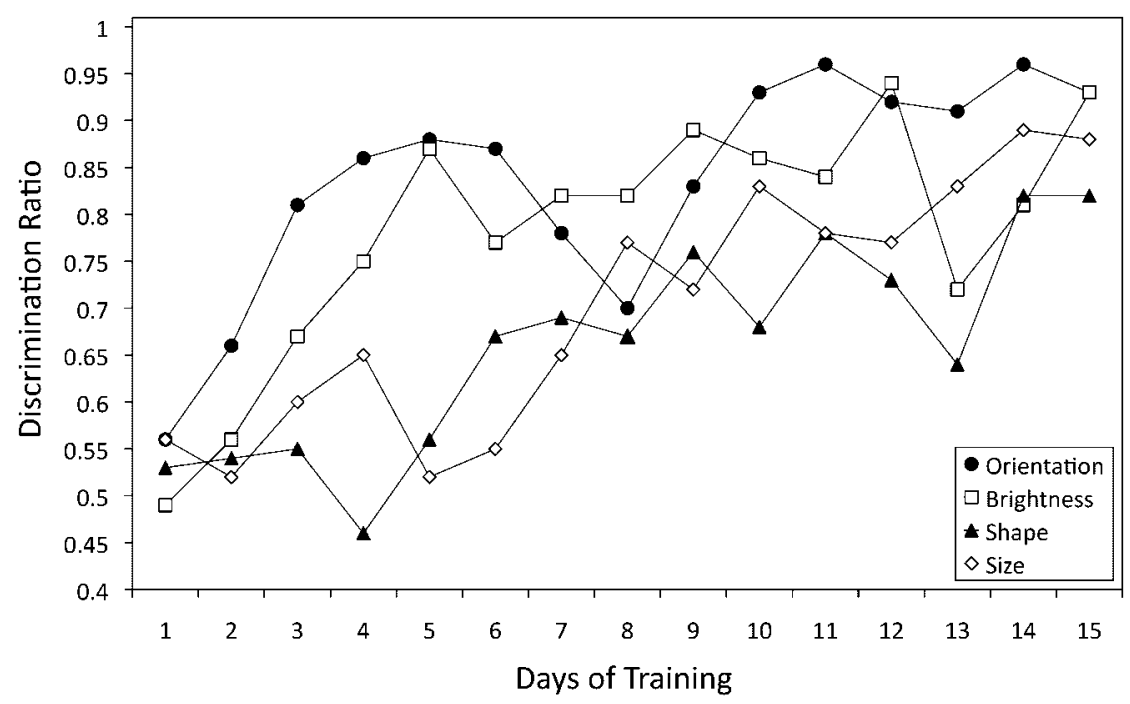

SSVL+ P7

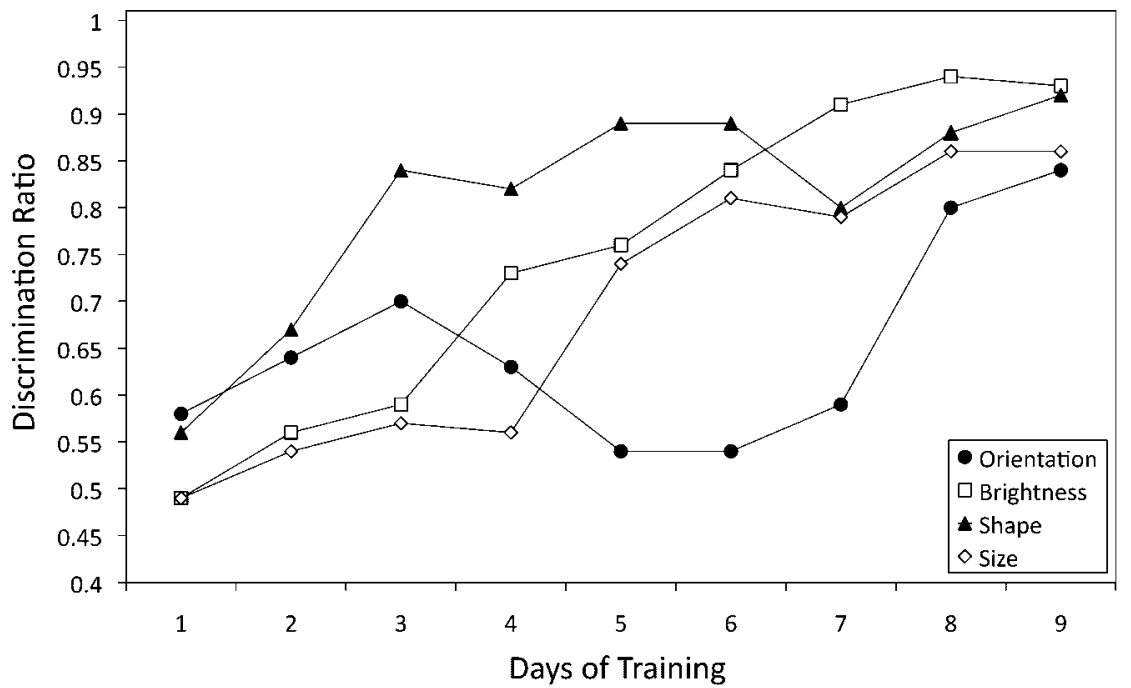

Fig. 3. Discrimination Ratios (DRs) for Shape, Size, Orientation, and Brightness for P21 (top) and P7 (bottom) throughout the entirety of Discrimination Training.

tradeoffs had or had not occurred. Further, it is difficult to fashion null hypothesis tests to determine whether or not any of those paired dimensional correlations-high or low-differ reliably from those that would be expected by chance alone.

With due regard to these statistical concerns, our pigeons were increasingly likely to exhibit low DR correlations the longer it took them to learn the MNC discrimination. Indeed, considering all nine birds, the correlation between the number of days of Discrimination Training needed to attain criterion performance and a pigeon's mean overall interdimensional correlation score was strongly negative, $r=-.88$, $p<.01$.

Comparing the functions depicted in Figures 2-4 with the correlations enumerated in Table 5 

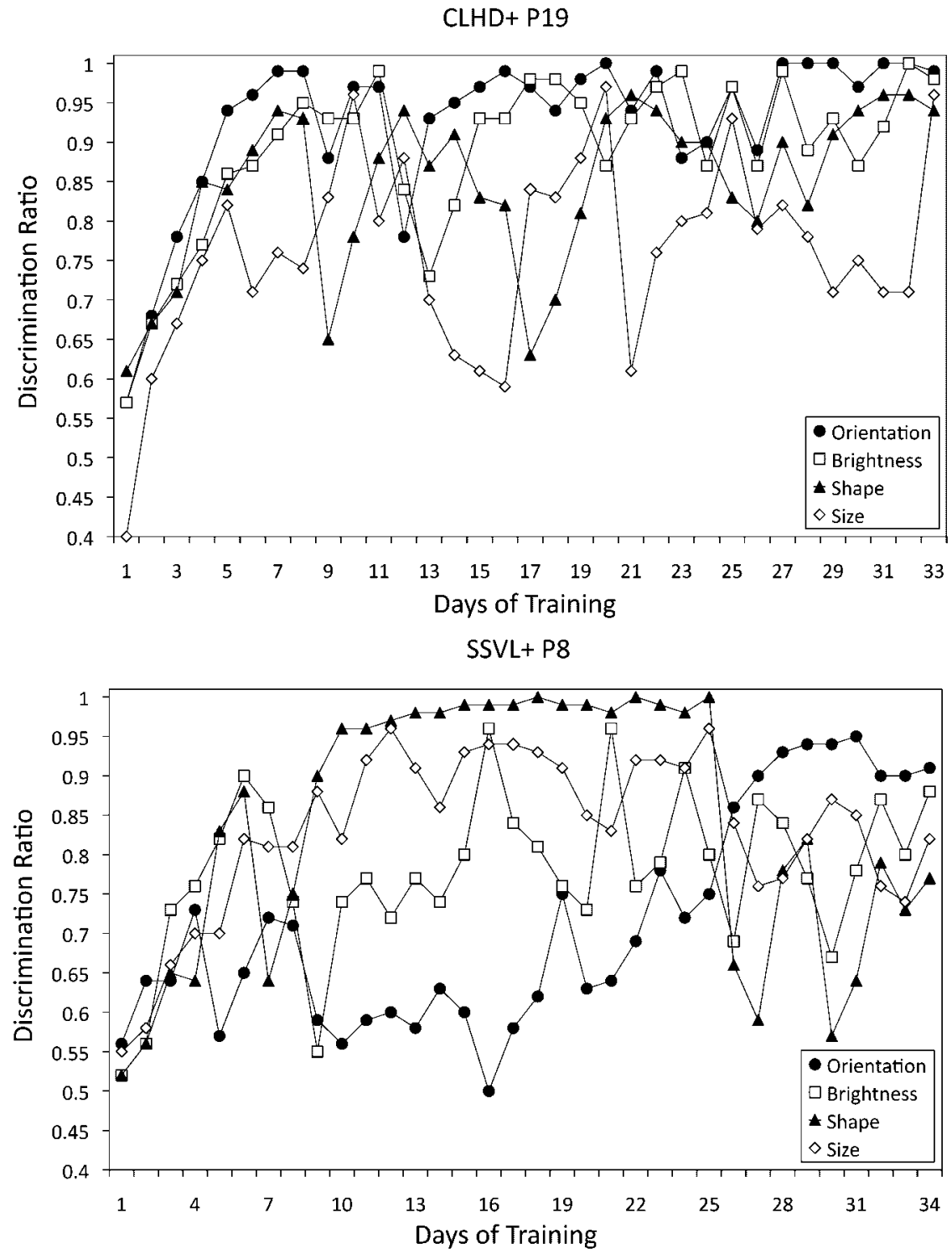

Fig. 4. Discrimination Ratios (DRs) for Shape, Size, Orientation, and Brightness for P19 (top) and P8 (bottom) throughout the entirety of Discrimination Training.

suggests that, among our six focal pigeons, slower learning was indeed accompanied by both more pronounced dimensional discrimination reversals and lower interdimensional correlations. Figure 4 shows that the pair of pigeons that were slowest to learn the four-dimensional discrimination, and that exhibited most of the lowest mean intercorrelation scores, also displayed the most striking attentional tradeoffs. Figure 3 illustrates more modest attentional tradeoffs by a pair of birds that exhibited a few low intercorrelation scores and that learned the four-dimensional discrimination at an intermediate speed. And, Figure 2 depicts only slight evidence of attentional tradeoffs by the pair of birds that exhibited most of the highest intercorrelation scores and that were fastest to learn the four-dimensional discrimination. This overall pattern of responding is just what would have been expected if our four-dimensional 
Table 5

Pairwise Correlations among All Four Dimensional DRs

\begin{tabular}{|c|c|c|c|c|c|c|c|c|c|}
\hline \multirow[b]{4}{*}{ Dimensional Pairing } & \multicolumn{9}{|c|}{ Correlation Coefficient } \\
\hline & \multicolumn{9}{|c|}{ Bird (Days of Training) } \\
\hline & \multicolumn{5}{|c|}{ CLHD+ Condition } & \multicolumn{4}{|c|}{ SSVL+ Condition } \\
\hline & P20 (7) & P21 (15) & P10 (20) & P17 (23) & P19 (33) & P9 (5) & P7 (9) & P11 (9) & P8 (34) \\
\hline Shape \& Size & 0.91 & 0.75 & 0.33 & 0.64 & 0.20 & 0.82 & 0.77 & 0.88 & 0.76 \\
\hline Shape \& Orientation & 0.88 & 0.55 & 0.55 & 0.08 & 0.56 & 0.77 & 0.32 & 0.72 & -0.46 \\
\hline Shape \& Brightness & 0.94 & 0.67 & 0.79 & 0.63 & 0.39 & 0.95 & 0.78 & 0.86 & 0.36 \\
\hline Size \& Orientation & 0.87 & 0.61 & 0.65 & 0.30 & 0.47 & 0.89 & 0.33 & 0.53 & -0.09 \\
\hline Size \& Brightness & 0.82 & 0.57 & 0.55 & 0.64 & 0.56 & 0.90 & 0.95 & 0.93 & 0.37 \\
\hline Orientation \& Brightness & 0.67 & 0.74 & 0.78 & 0.68 & 0.81 & 0.79 & 0.38 & 0.61 & 0.14 \\
\hline Mean & 0.85 & 0.65 & 0.61 & 0.50 & 0.50 & 0.85 & 0.59 & 0.76 & 0.18 \\
\hline
\end{tabular}

discrimination task were taxing the attentional limits of at least some of our pigeons (please consult the Supplementary Electronic Material for data for the other three pigeons).

\section{Attentional Tradeoffs: Response Rates}

Although it is surely appropriate to focus on the pigeons' dimensional DR scores to assess discriminative performance on the MNC task, it must be appreciated that these scores are derived from responding on both $\mathrm{S}+$ and $\mathrm{S}-$ trials. Therefore, we next examined the birds' responding to all 16 compound discriminative stimuli throughout training. Plotting all 16 stimuli on the same coordinates proved to be too chaotic; so, we separated each of the nine pigeons' responding into four separate plots, one for each dimensional disparity (DD1, DD2, DD3, and DD4). These sets of functions are shown in the Supplementary Electronic Material. Scrutiny of these scores revealed that most of the attentional tradeoffs (such as those) depicted in Figures 2-4 arose from responding on DD1 trials; therefore, these DD1 scores for the six focal pigeons are shown in Figures 5-7.

Three points can be made about these graphs. First, responding to the $\mathrm{S}+$ was initially clustered together with the S- compounds, but ultimately surpassed all of them by a wide margin. Second, upward and downward swings in the pigeons' rates of $S$ - responding were also evident, with more numerous and extreme swings being associated with slower discrimination learning. Third, changes in the pigeons' rates of responding to the $\mathrm{S}+$, although present, did not accord with upward and downward swings in the pigeons' rates of responding to the S-s.
Careful comparison of Figures 2, 3, and 4 with Figures 5, 6, and 7, respectively, reveals that most of the upward and downward swings in the pigeons' DRs were accompanied by coordinated downward and upward swings in their rates of Sresponding. Still, there were a few exceptions, which can be seen in the case of the slowest learning pair of birds (compare Figs. 4 and 7). Although P19 exhibited Size-Shape tradeoffs in DRs on Days 17, 21, and 25, no clear response rate tradeoff was evident on Day 25. And, although P8 showed Brightness-Orientation tradeoffs in DRs on Days 16, 19, and 21, no clear response rate tradeoff was seen on Day 16. Such exceptions were relatively rare and unsurprising; remember that the DRs depicted in Figures 2, 3, and 4 were derived from responding to all 16 discriminative stimuli, whereas the S- response rate scores depicted in Figures 5, 6, and 7 involved only 4 of those 16 stimuli.

\section{Discussion}

\section{Empirical Synopsis}

Mastering a complex task that requires the discrimination of stimuli from four simultaneously presented visual dimensions would be expected to tax the attentional capacities of most animals. Yet, all nine of our pigeons eventually acquired the four-dimensional MNC discrimination to very high levels of accuracy in a mean of only 17 days of Discrimination Training (range: 5-34 days; Tables 1 and 2).

Overall, the four visual dimensions-Shape, Size, Orientation, and Brightness-from which we constructed the 16 compound discriminative stimuli proved to be of roughly similar salience, 

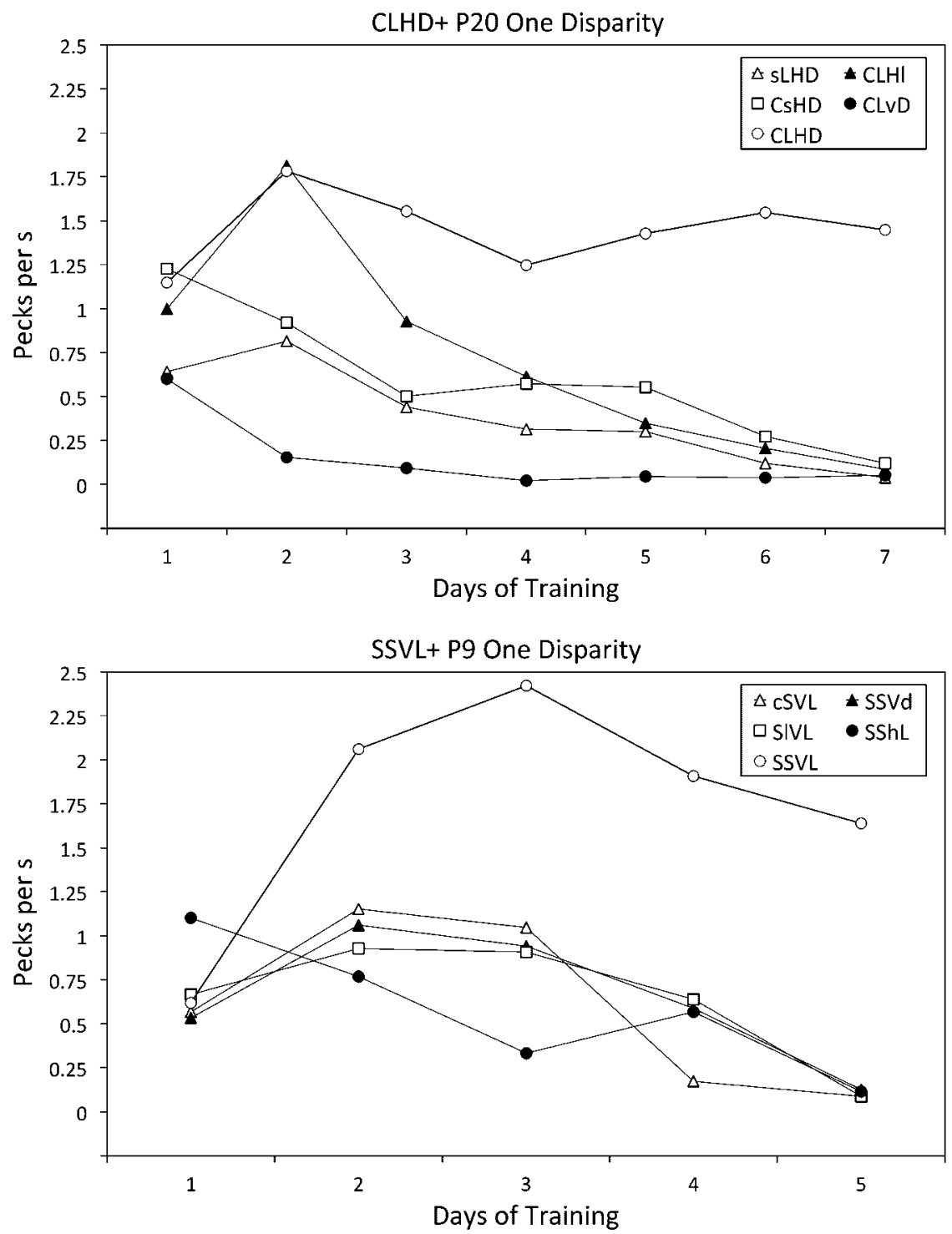

Fig. 5. Mean daily rate of responding to $\mathrm{S}+$ and to each of the four discriminative stimuli that differed from $\mathrm{S}+$ along a single dimension (DD1) for P20 (top) and P9 (bottom). Lower case letters indicate that the S- differed from the S+ along that dimension: Shape (Circle/Square), Size (Large/Small), Orientation (Horizontal Line/Vertical Line), and Brightness (Dark/Light).

although individual pigeons came under the control of some dimensions a bit more quickly or strongly than others (Table 3; Figs. 2-4). In addition, the greater the number of dimensions along which the S-s differed from the $\mathrm{S}+$, the easier was the discrimination; compared with the four-dimensional disparity $S$-, the one-dimensional disparity S-s proved to be the most difficult to discriminate from the $\mathrm{S}+$ and the two-dimen- sional disparity S-s proved to be the next most difficult to discriminate from the $\mathrm{S}+$ (Table 4).

The most interesting and challenging of our findings involved the tradeoffs in discriminative responding that our pigeons exhibited during the course of Discrimination Training (Table 5; Figs. 2-7). Three trends were especially noteworthy in this regard. First, considering the discrimination learning scores of our pigeons, 

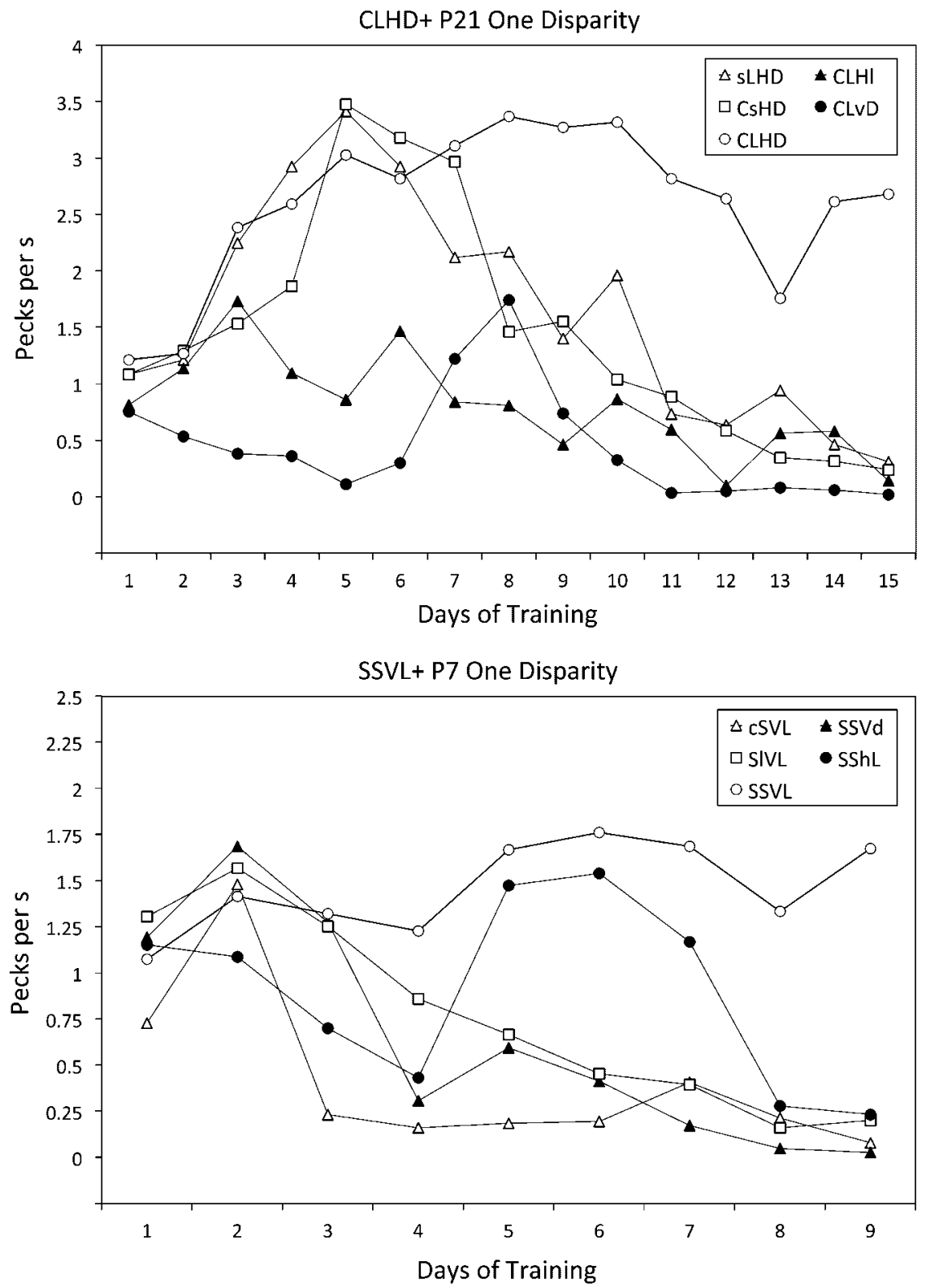

Fig. 6. Mean daily rate of responding to $\mathrm{S}+$ and to each of the four discriminative stimuli that differed from $\mathrm{S}+$ along a single dimension (DD1) for P21 (top) and P7 (bottom). Lower case letters indicate that the S- differed from the S+ along that dimension: Shape (Circle/Square), Size (Large/Small), Orientation (Horizontal Line/Vertical Line), and Brightness (Dark/Light).

the longer it took the birds to learn, the more likely they were to exhibit such tradeoffs. Second, some but not all of the dimensions participated in such dimensional tradeoffs. Third, pigeons' response rate scores to the compound discriminative stimuli closely ac- corded with their DR scores (cf. Figs. 2-4 to Figs. 5-7). Upward and downward swings in the birds' DRs were respectively accompanied by downward and upward swings in their rate of Sresponding, but much less so by variations in their rate of $\mathrm{S}+$ responding. 

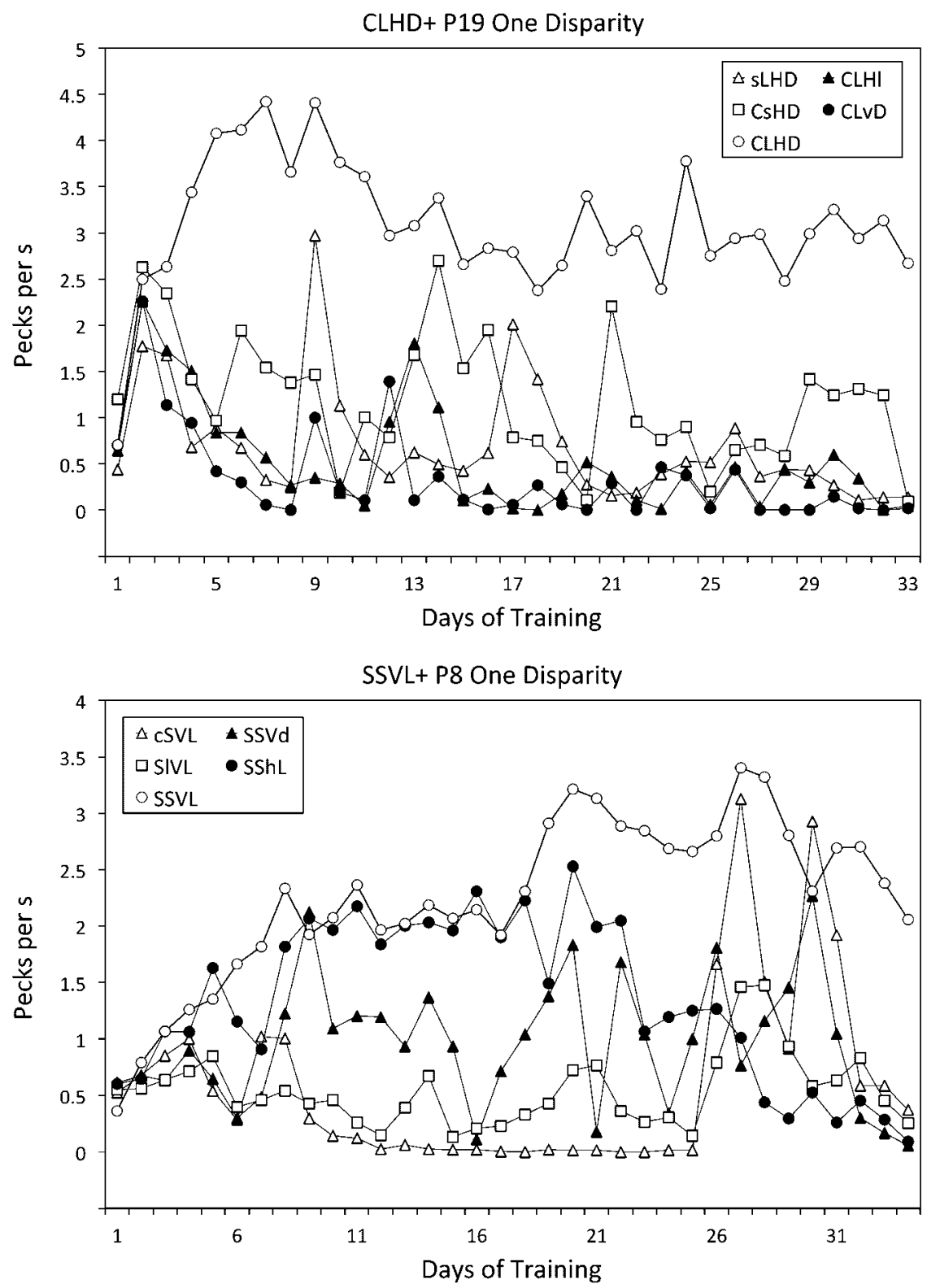

Fig. 7. Mean daily rate of responding to $\mathrm{S}+$ and to each of the four discriminative stimuli that differed from $\mathrm{S}+$ along a single dimension (DD1) for P19 (top) and P8 (bottom). Lower case letters indicate that the S- differed from the S+ along that dimension: Shape (Circle/Square), Size (Large/Small), Orientation (Horizontal Line/Vertical Line), and Brightness (Dark/Light).

\section{Selective Attention}

Most theories of attention in discrimination learning (Kruschke \& Johansen, 1999; Pashler, 1998; Riley \& Roitblat, 1978; Sutherland \& Mackintosh, 1971; Thomas, 1970; Trabasso \& Bower, 1968) propose that the tradeoffs in discriminative responding that our pigeons displayed were likely to have arisen from the birds' limited capacity to simultaneously process all four dimensions of the compound visual stimuli prior to discrimination mastery. Such attentional tradeoffs can be deemed to follow from two key aspects of attention: limited 
capacity and selectivity. So, when the S-s differed from the $\mathrm{S}+$ in only a single way, there was no redundancy in the discriminative dimensions that were available to guide the pigeons' pecking behavior; all four dimensions had to be processed, thereby leaving some birds to struggle in discriminating one or two of the visual dimensions. This pattern of performance, although in general accord with the inverse hypothesis of attentional theories of discrimination learning, cannot readily answer the following important and challenging questions.

Why did some pigeons learn quickly and show little evidence of attentional tradeoffs, whereas other pigeons learned slowly and showed strong evidence of attentional tradeoffs? Why might some of the birds have begun to discriminate all four dimensions early in training, only to have their performance to one or more of the dimensions subsequently fall? Why did some dimensions, but not others appear to reciprocally interact with one another? Why might those interacting dimensions have been different for different pigeons? (Relatedly, Reynolds, 1961, Experiment 1, was unable to say why one of his pair of birds attended to shape rather than to color, whereas the other bird attended to color rather than to shape.) Perhaps varying the discriminability of the stimuli along the visual dimensions or giving prior discrimination training along one or more of the dimensions will help provide answers, much as the study of overshadowing and blocking advanced the development of associative learning theories.

\section{Selective Association}

The prime interpretive rival to stimulus selection phenomena in discrimination learning derives from research into so-called "selective associations." Here, entirely non-attentional, associative learning theories have been proposed to explain such phenomena as overshadowing and blocking. Indeed, these associative theories have largely held sway over attentional interpretations in recent years. Readers might thus reasonably ask: Can a nonattentional, associative learning theory explain the many details of our pigeons' discrimination behavior? We attempted to answer this question, appreciating that there might be other accounts of our findings.

Although there are many competing theories of associative learning (Wasserman \& Miller, 1997), we chose the Rescorla-Wagner (1972) model as our benchmark because of its widespread acceptance and influence. We examined a broad range of parameters across many simulations in an effort to assess the viability of this model. In the interests of concision, we report the results inspired by the behavior of P19. This bird had taken a long 33 days to meet criterion (Tables 1 and 2); it had shown a pattern of dimensional stimulus control that matched the group mean (Table 3); it had displayed a pattern of discrimination behavior to increasingly discrepant S-s that matched the group mean (Table 4); and, it had exhibited dramatic tradeoffs in dimensional discrimination behavior (Table 5, Figs. 4 and 7).

To apply the model (using the R\&W Simulator of Alonso, Mondragón, \& Fernández, 2012), we used only four dimensional salience values to predict the pattern of P19's responding to all 16 of the compound discriminative stimuli. We picked those four salience parameter values from the pattern of responding that the pigeon had exhibited to each dimension averaged across the other three dimensions (Table 3). We contented ourselves with four parameter values which yielded associative strength scores which plausibly accorded with the bird's data, but we did not attempt to determine the best parameter values. We were merely engaged in a "proof of concept" simulation.

Given salience parameters of .200 for Orientation, .100 for Brightness, .050 for Shape, and .025 for Size, we generated associative strength scores for each of the 16 compound discriminative stimuli. Other parameter valueswere: learning rate on reinforced trials $($ beta +$)=.120$, learning rate on nonreinforced trials (beta-) $=.110$, asymptote of associative strength on reinforced trials $($ lambda +$)=1.000$, asymptote of associative strength on nonreinforced trials (lambda-) $=.000$, and the number of training trials with each compound stimulus $=80$. Comparing the resulting theoretical scores with the pigeon's actual rates of response to all 16 stimuli averaged across all 33 days of Discrimination Training (Table 1) yielded a respectable correlation of .73.

We also calculated the absolute numerical disparities in associative strength between P19's $\mathrm{S}+$ and each of its $15 \mathrm{~S}$-s (because negative associative strength scores make it impossible to calculate DRs) and we then used those scores to predict the bird's discriminative responding to the four individual dimensions (Table 3 ) and 
to the four different disparities between $\mathrm{S}+$ and different groupings of S-s (Table 4); the former correlation was .99 and the latter was .87 .

Although the original Rescorla-Wagner model effectively reproduced the overall patterns of discriminative responding by $\mathrm{P} 19$, we added one more wrinkle to our simulation exercise: We included a unique configural element to each of the 16 four-dimensional compound stimuli (Rescorla, 1973; Whitlow \& Wagner, 1972). It is, after all, entirely plausible to expect some degree of configural stimulus control in our MNC task; this task bears some resemblance to Pavlovian Positive Patterning, which also permits solution by both elemental and configural stimuli (Wasserman \& Miller, 1997).

We chose a salience value for the 16 configural stimuli of .200 (still higher salience values sped discrimination learning and lessened the effect of dimensional control). Comparing those theoretical associative strength scores involving the configural stimulus parameter with the pigeon's actual rate of response to all 16 stimuli averaged across all 33 days of Discrimination Training (Table 1) yielded a correlation of .96. This marked improvement in the model's predictive power from .73 without configural stimuli to .96 with configural stimuli does not by itself confirm the participation of configural learning in our task, but it is consistent with that possibility (the reason for this marked improvement is detailed below).

Of course, the key matter of interest was not the overall level of discrimination behavior that the Rescorla-Wagner model predicted, but rather the dynamics of discrimination learning: Can it explain the session-to-session yin and yang of dimensional stimulus control exhibited by P19 and other pigeons? The answer here was an unequivocal and unsurprising, "No." Figure 8 (top) reproduces P19's discrimination learning performance accompanied by its simulated performance without configural stimuli (middle) and with configural stimuli (bottom). Both sets of acquisition functions are smooth and negatively accelerated, lacking any hint of tradeoffs, completely unlike P19's actual behavior and mathematically required by linear operator models like the Rescorla-Wagner model.

The failure of the Rescorla-Wagner model to reproduce those tradeoffs was not because it did not faithfully reproduce discrimination learning that was properly ordered in terms of dimen-
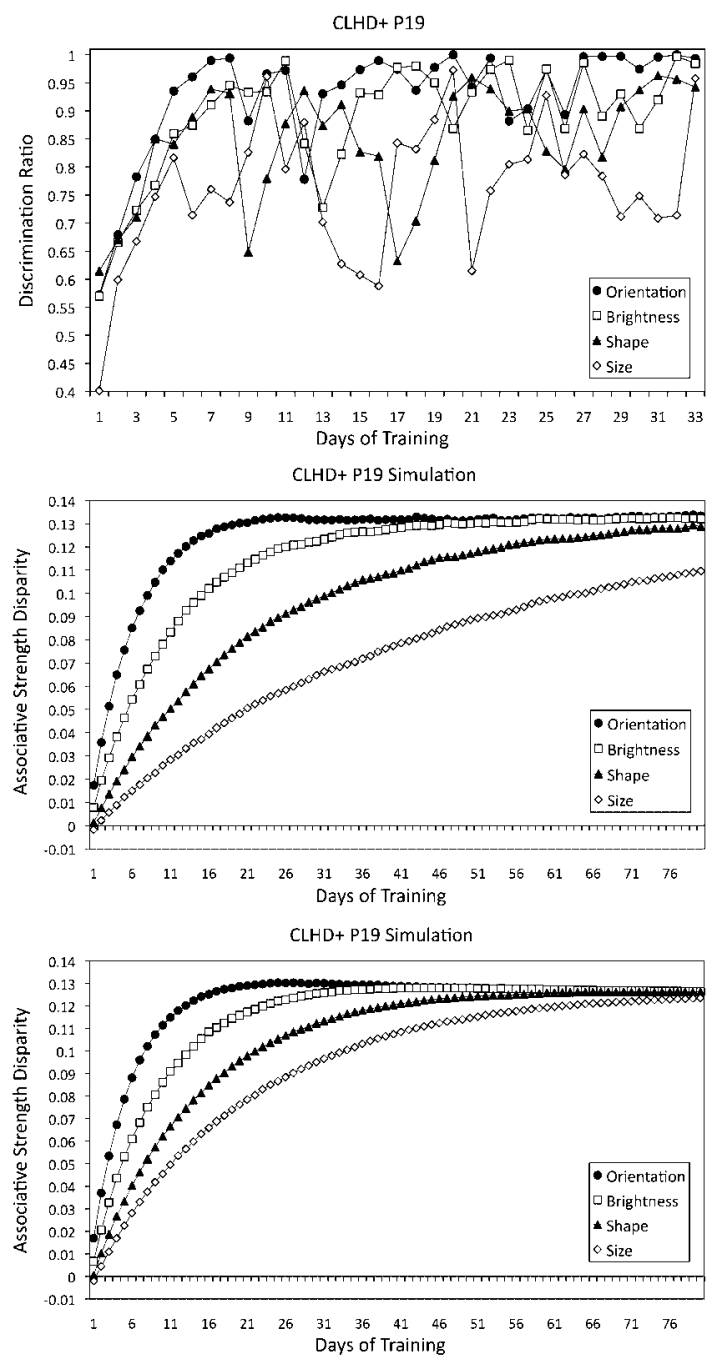

Fig. 8. Comparisons of real and simulated pigeon discrimination learning. Top: Discrimination Ratios (DRs) for Shape, Size, Orientation, and Brightness for P19 throughout the entirety of Discrimination Training. Middle: P19's simulated discrimination learning performance without configural stimuli along the dimensions of Shape, Size, Orientation, and Brightness. Bottom: P19's simulated discrimination learning performance with configural stimuli along the dimensions of Shape, Size, Orientation, and Brightness (bottom). See text for details concerning these simulations.

sional salience; it did so quite well both without and with configural stimuli (Fig. 8). Nor did the model fail to capture the orderly unfolding of discrimination learning in terms of the number of dimensions along which the S-s differed from the S+. Figure 9 (top) illustrates P19's actual 

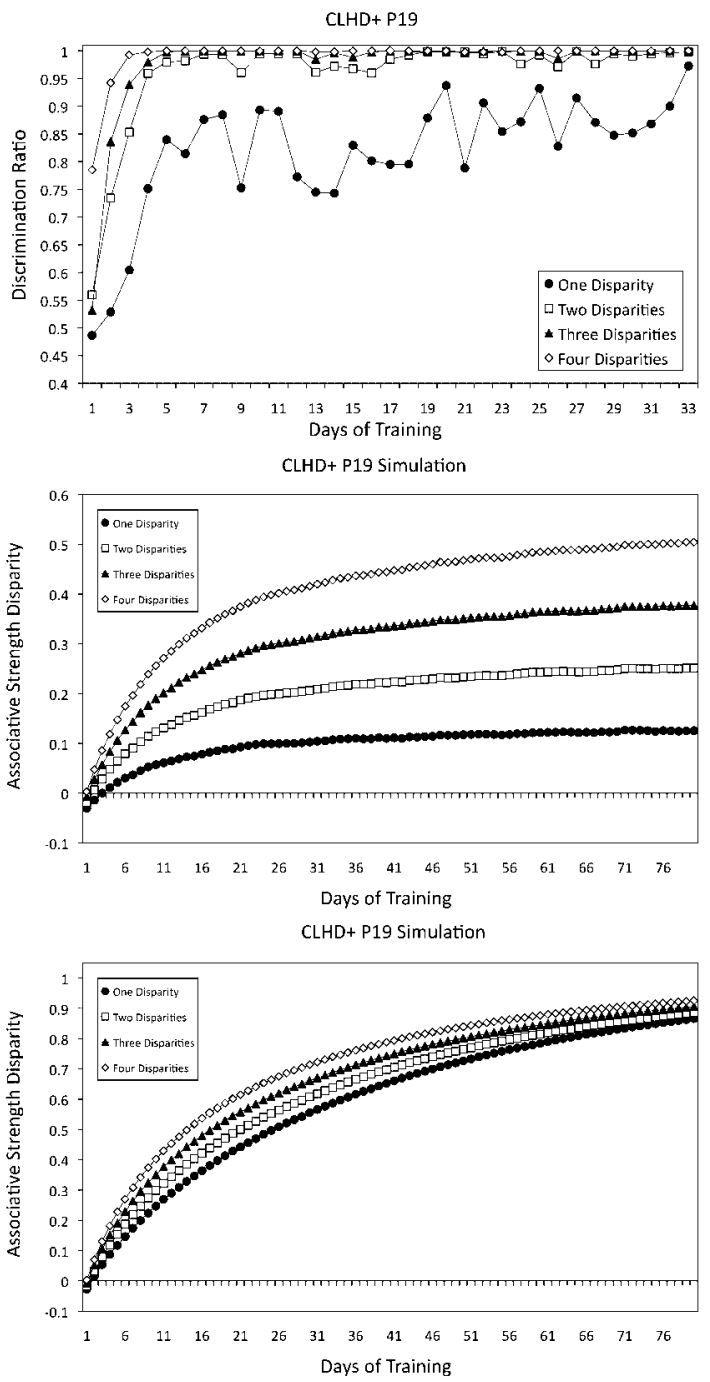

Fig. 9. Comparisons of real and simulated pigeon discrimination learning. Top: Discrimination Ratios (DRs) for S-s that differed from S+ along one, two, three, or four dimensions for P19 throughout the entirety of Discrimination Training. Middle: P19's simulated discrimination learning performance without configural stimuli for S-s that differed from $\mathrm{S}+$ along one, two, three, or four dimensions. Bottom: P19's simulated discrimination learning performance with configural stimuli for S-s that differed from $\mathrm{S}+$ along one, two, three, or four dimensions (bottom). See text for details concerning these simulations.

DRs throughout Discrimination Training as a function of the number of ways in which the S-s differed from the $\mathrm{S}+$; the same ordering is seen in the theoretical dimensional disparity scores (calculated from the associative strength scores of the 16 compound stimuli) in Figure 9 (middle) without configural stimuli and in Figure 9 (bottom) with configural stimuli.

Critically, Figure 9 (bottom) shows that the configural simulation more faithfully captured the converging empirical discrimination scores (Fig. 9, top) for all four disparities between $\mathrm{S}+$ and the S-s toward the end of Discrimination Training than did the nonconfigural simulation. Indeed, the nonconfigural simulation (Fig. 9, middle) erroneously predicted progressively diverging scores, as well as progressively lower asymptotic discrimination performance to S-s differing from $\mathrm{S}+$ along four, three, two, or one dimension(s). Our simulation thus provides critical mathematical evidence pointing to the participation of configural cues in MNC discrimination learning; future research should seek confirmatory experimental evidence to support this possibility.

\section{Selective Attention versus Selective Association}

Were it not for its failure to account for the dynamic discriminative tradeoffs that our pigeons exhibited, the most prominent associative learning theory (specifically, the "configural" Rescorla-Wagner model) might have provided a satisfying account of our pigeons' interesting pattern of discrimination learning. So too might other "configural" accounts of discrimination learning (Pearce, 2002; Wasserman \& Miller, 1997). However, all of these accounts predict monotonic changes in discriminative responding, unlike the correlated upshifts and downshifts in performance that our pigeons evidenced, also arguing against "hybrid" accounts which hypothesize that pigeons might first engage in dimensional stimulus processing before progressing to configural stimulus processing.

So, we are left with the rather unsatisfying state of affairs that theories of selective attention descriptively comport with the discriminative tradeoffs shown by our pigeons, but they lack the rigor and explicitness of associative learning accounts. Nonattentional, associative learning theories succeed in accounting for the overall pattern of results, but they fail to capture the dynamic tradeoffs that here proved to be so interesting and challenging.

Faced with this state of affairs, we plan future investigations in which other conditions of training-for instance, two-alternative, forcedchoice discrimination training-might help elucidate the role of selective attention in 
pigeons' four-dimensional discrimination behavior. In this case, perhaps fewer demands would be placed on the birds' possibly limited attentional and associative resources.

Whatever the outcome of that future research, we believe that the MNC task affords unique experimental and theoretical opportunities for advancing our understanding of discrimination learning. The role of selective attention, the interplay between elemental and configural stimulus control, and the assessment of information processing capacity can all be explored with this promising discrimination methodology.

\section{References}

Alonso, E., Mondragón, E., \& Fernández, A. (2012). A Java simulator of Rescorla and Wagner's prediction error model and configural cue extensions. Computer Methods and Programs in Biomedicine, 108, 346-355.

Blough, D. S., \& Blough, P. M. (1997). Form perception and attention in pigeons. Animal Learning $\mathcal{E} \mathcal{E}$ Behavior, 25, 1 20.

Castro, L., \& Wasserman, E. A. (2013). Information-seeking behavior: Exploring metacognitive control in pigeons. Animal Cognition, 16, 241-254.

Chatlosh, D. L., \& Wasserman, E. A. (1993). Multidimensional stimulus control in pigeons: Selective attention and other issues. In T. R. Zentall (Ed.), Animal cognition: A tribute to Donald A. Riley (pp. 271-292). Hillsdale, NJ: Erlbaum.

Farthing, G. W., \& Hearst, E. (1970). Attention in the pigeon: Testing with compounds or elements. Learning and Motivation, 1, 65-78.

Gottselig, J. M., Wasserman, E. A., \& Young, M. E. (2001). Attentional tradeoffs in pigeons learning to discriminate newly-relevant visual stimulus dimensions. Learning and Motivation, 32, 240-253.

Johnson, D. F., \& Cumming, W. W. (1968). Some determiners of attention. Journal of the Experimental Analysis of Behavior, 11, 157-166.

Kruschke, J. K., \& Johansen, M. K. (1999). A model of probabilistic category learning. Journal of Experimental Psychology: Learning, Memory, and Cognition, 25, 1083-1119.

Lovie, A. D. (1983). Attention and behaviourism-fact and fiction. British Journal of Psychology, 74, 301-310.
Pashler, H. E. (1998). The psychology of attention. Cambridge, MA: MIT Press.

Pearce, J. M. (2002). Evaluation and development of a connectionist theory of configural learning. Animal Learning Eै Behavior, 30, 73-95.

Rescorla, R. A. (1973). Evidence for the "unique stimulus" account of configural conditioning. Journal of Comparative and Physiological Psychology, 85, 331-338.

Rescorla, R. A., \& Wagner, A. R. (1972). A theory of Pavlovian conditioning: Variations in the effectiveness of reinforcement and nonreinforcement. In A. H. Black, \& W. F. Prokasy (Eds.), Classical conditioning II: Current theory and research (pp. 64-99). New York: Appleton-CenturyCrofts.

Reynolds, G. S. (1961). Attention in the pigeon. Journal of the Experimental Analysis of Behavior, 4, 203-208.

Riley, D. A., \& Roitblat, H. L. (1978). Selective attention and related cognitive processes in pigeons. In S. H. Hulse, H. Fowler, \& W. K. Honig (Eds.), Cognitive processes in animal behavior (pp. 249-276). Hillsdale, NJ: Erlbaum.

Soto, F. A., \& Wasserman, E. A. (2010). Integrality/ separability of stimulus dimensions and multidimensional generalization in pigeons. Journal of Experimental Psychology: Animal Behavior Processes, 36, 194-205.

Soto, F. A., \& Wasserman, E. A. (2011). Asymmetrical interactions in the perception of face identity and emotional expression are not unique to the primate visual system. Journal of Vision, 11, 1-18.

Sutherland, N. S., \& Mackintosh, N. J. (1971). Mechanisms of animal discrimination learning. New York: Academic Press.

Thomas, D. R. (1970). Stimulus selection, attention, and related matters. In J. H. Reynierse (Ed.), Current issues in animal learning (pp. 311-356). Lincoln, NE: University of Nebraska Press.

Trabasso, T., \& Bower, G. H. (1968). Attention in learning. New York: Wiley.

Wasserman, E. A., \& Miller, R. R. (1997). What's elementary about associative learning? Annual Review of Psychology, 48, 573-607.

Whitlow, J. W., Jr., \& Wagner, A. R. (1972). Negative patterning in classical conditioning: Summation of response tendencies to isolable and configural components. Psychonomic Science, 27, 299-301.

Wilkie, D. M., \& Masson, M. E. (1976). Attention in the pigeon: A reevaluation. Journal of the Experimental Analysis of Behavior, 26, 207-212.

Received: June 25, 2013

Final Acceptance: February 19, 2014 